\title{
An Improved Version of the Multi-Attribute Task Battery (MATB) and Data Reduction Program (MATPROC)
}

\author{
Scott $\mathrm{H}$. Mills and Kirby Gilliland \\ Department of Psychology, University of Oklahoma, Norman, OK 73019-0535
}

This demonstration will present both an improved version of the Multi-Attribute Task Battery (MATB; Comstock \& Arnegard, 1991) that provides markedly better sound capability while eliminating the need for a second microcomputer, and an associated user-friendly data reduction and processing package called MATPROC.

Multi-Attribute Task Battery. The MATB was developed at NASA Langley Research Center to provide a comprehensive behavioral metric for assessing complex operator performance. The MATB includes a Monitoring task that consists of both a set of response time stimuli and a set of probability monitoring dials, a compensatory Tracking task, a Resource Management task that is presented as a fuel tank management task, and an auditory Communications task patterned after a radio dial setting task. The battery is actually designed to be implemented as a complex cognitive task not unlike a synthetic work task. Given the selection and structure of the tasks, the MATB has strong similarity to an aircrew operations environment. The user has the ability to present any of the tasks singly, in combination, or all of the tasks simultaneously. A user-friendly script system provides the experimenter with a high degree of control over the scheduling of subtask onset and offset. The auditory Communications task and the Resource Management task are unique features of this battery. Another unique feature is that the MATB can be paused at any time for on-screen presentation of the NASA-TLX subjective workload scale-a helpful feature for those who want concomitant subjective workload ratings. The MATB runs on a 286,386 , or $486 \mathrm{PC}$ with $4 \mathrm{Mb}$ RAM, EGA graphics, and a mouse or joystick. Current work is under way at the Personality Research Laboratory at the University of Oklahoma and at the Human Performance Branch of Armstrong Laboratory at WrightPatterson AFB using the MATB as a complex cognitive task in explorations of cognitive psychophysiology, sustained operations, and stress/adaptation. A number of other university and government research laboratories are exploring the use of MATB as well.

Despite its obvious attractive features, use of the MATB presents two major problems. To implement the Communications task requires an additional yoked microcomputer equipped with a sound board, and the resulting audio messages are not of high voice quality. The second problem is the volume of data that the MATB produces. Each session produces five raw data files, and these files can be extensive if trial length is long and/or stimulus frequency is high.

One purpose of this demonstration is to present a new version of MATB that incorporates a single sound card eliminating the need for a second microcomputer and producing high voice quality for the Communications task. This version successfully integrates a SoundBlaster card with the MATB. The user can record selected Communications Task messages and then access those messages at will through MATB script commands any time in the MATB test session. Simple variations of this feature can be utilized for a variety of alternative auditory vigilance tasks.

$M A T P R O C$. The second major problem with MATB is addressed by MATPROC. MATPROC is an integrated set of macros written for Microsoft Excel that provides a comprehensive system for processing and consolidating raw MATB data files. MATPROC is menu driven and user friendly. It allows the user to specify the raw data files to process. The user can select any single subtask raw data file for any specific trial, or all of the subtask raw data files for multiple trials. The user can select from among several dependent variables for each subtask. In addition, the user can process the overall trial length by a variety of epoch lengths. For example, if the MATB trial was 30 minutes long, a user could specify the data be parceled and reduced in $30 \mathrm{sec}$ epochs, 5 minute epochs, 15 minute epochs, and an overall 30 minute epoch-all within the same analysis run. The user can also specify output file characteristics. Output files are easily imported into spreadsheets, SAS, SPSS, or mainframes. MATPROC will analyze individual files or can batch process numerous files. We have successfully processed thousands of files in single runs. This capability reduces several days or weeks of data analysis into a few hours. New features are being added to MATPROC such as trim capability so the user can trim data at the beginning or end of the MATB trial.

\section{REFERENCES}

Comstock, J. R., \& Amegard, R. J. (1991). The Multi-Attribute Task Battery for human operator workload and strategic behavior research. NASA TM104174.

-Direct inquiries to address above or (405) $325-4511$ or to: KIRBY@OUPSY.PSY.UOKNOR.EDU. 